Les Archives Suisses de Médecine Vétérinaire (ASMV), la revue de la Société des Vétérinaires Suisses (SVS), célèbrent cette année leur 200e anniversaire. En comparaison, le Bulletin des médecins suisses fait figure de "novice» du haut de ses 96 ans. Au nom de la FMH, je tiens à adresser toutes mes félicitations à la SVS pour le bicentenaire de sa revue qui est également la plus ancienne revue vétérinaire au monde. La SVS et la FMH collaborent aux côtés d'autres organisations médicales sur différents thèmes communs, dont l'hygiène des denrées alimentaires et la résistance aux antibiotiques. L'approche "One Health" développée à cet effet le montre bien: c'est ensemble que médecins et vétérinaires doivent aborder les enjeux nationaux et internationaux comme la propagation des bactéries résistantes aux antibiotiques.

Dr Jürg Schlup, président de la FMH

\title{
Pour le progrès politique et scientifique
}

\section{Annik Steiner}

Responsable Communication et médias, Société des Vétérinaires Suisses (SVS)

Les Archives Suisses de Médecine Vétérinaire (ASMV) célèbrent cette année leur 200 anniversaire. A l'origine, la plus ancienne revue vétérinaire au monde était publiée conjointement aux «Archives de la médecine, chirurgie et pharmacie». Récemment, les médecins et les vétérinaires ont renouvelé et renforcé leur collaboration afin de défendre ensemble leurs intérêts communs.

Fondée en 1813, la Société des Vétérinaires Suisses (SVS) est l'une des premières associations nationales, marquée alors par le souci d'assurer une lutte d'envergure nationale contre les épizooties. La génération des jeunes universitaires réclamait du progrès dans les acquis démocratiques et dans la recherche scientifique, et s'intéressait de plus en plus au libéralisme et à la liberté de pensée. C'est à des fins de diffusion des nouvelles connaissances qu'ils ont créé des organes de publication. Un certain nombre de revues dédiées au progrès politique ou scientifique étaient ainsi publiées par Heinrich Remigius Sauerländer à Aarau. C'est vers ces éditions également que se sont tournés les deux médecins Ignaz Paul Vital Troxler, de Beromünster (1780-1866), et Franz Karl Stadlin, de Zoug (1777-1829). Le premier souhaitait publier des "Archives de la médecine, chirurgie et pharmacie», le second des "Archives de médecine vétérinaire». Les deux sont parues dans une mise en page identique dès 1816 auprès de Sauerländer. La publication médicale a cessé de paraître en 1817, alors que la publication vétérinaire fête aujourd'hui le bicentenaire de sa création. [1]

\section{Archives à destination des vétérinaires suisses}

Depuis 200 ans, les Archives Suisses de Médecine Vétérinaire (ASMV) rendent compte des connaissances et découvertes en médecine vétérinaire. Organe de

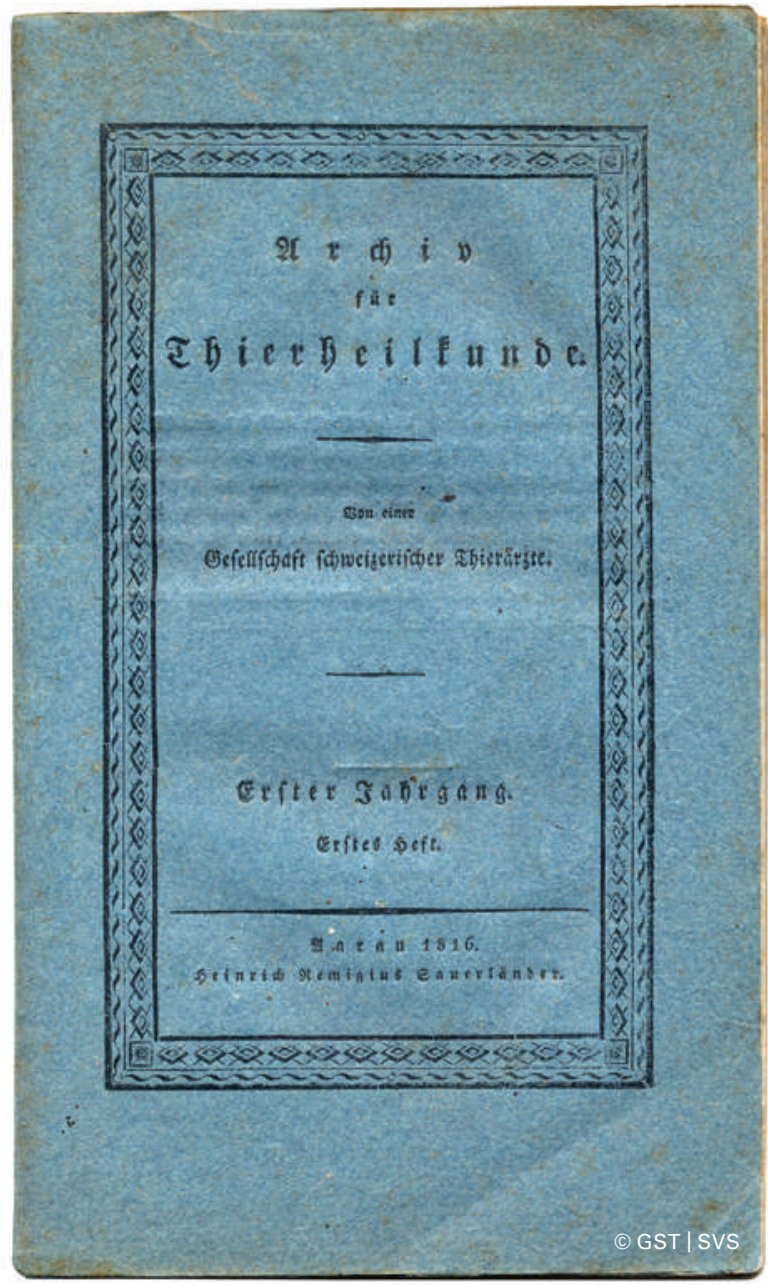


publication d'orientation scientifique, les ASMV permettent à leurs lecteurs d'échanger, mais aussi de se former. A l'échelle internationale, il s'agit du plus ancien périodique de médecine vétérinaire encore existant. Lorsque l'on ouvre les volumes des ASMV année

Lorsque l'on ouvre les volumes des ASMV année après année, on y lit et vit l'histoire de la médecine vétérinaire en Suisse.

après année, on y lit et vit l'histoire de la médecine vétérinaire en Suisse. Avec ce périodique, les ASMV constituent donc les archives de la SVS et offrent simultanément une image de l'histoire des vétérinaires suisses.

\section{Organe associatif et trait d'union avec la politique professionnelle}

Le Bulletin des médecins suisses (BMS) n'a vu le jour qu'environ 100 ans plus tard, en 1920. A l'instar du BMS pour la FMH, les ASMV de la SVS servent également d'organe associatif et permettent de faire le lien avec la politique professionnelle. Comme c'était le cas lors de la création de leurs publications scientifiques respectives, la médecine humaine et la médecine vétérinaire connaissent aujourd'hui à nouveau une collaboration accrue. En novembre 2015, le Conseil fédéral a adopté la stratégie nationale "Antibiorésistance» (StAR), conçue selon l'approche dite «One Health» (une seule santé) qui réunit médecine humaine, médecine vétérinaire et agriculture, et définit des objectifs et des mesures visant à préserver l'efficacité des antibiotiques pour l'homme et les animaux. Le succès des mesures prévues requiert l'engagement de tous les acteurs impliqués.
L'une des activités principales de la médecine vétérinaire est l'hygiène des denrées alimentaires. Le slogan "de l'étable à la table» conserve aujourd'hui encore toute sa pertinence. Les vétérinaires suivent les animaux de la naissance à l'abattage et surveillent le traitement des aliments d'origine animale aux côtés d'autres spécialistes, jusqu'à l'assiette du consommateur. En étant présent tout au long de la chaîne de production, le vétérinaire assure un produit final de haute qualité et d'une parfaite innocuité, contribuant ainsi à une nourriture saine et à une population en bonne santé.

\section{Le «Front bleu» pour davantage de poids politique}

Médecins et vétérinaires ont également renforcé leur collaboration avec la fondation l'année dernière du "Front bleu», qui réunit les chiropraticiens, les pharmaciens, les médecins-dentistes, la FMH et la SVS. Le Front bleu doit donner plus de poids politique aux intérêts communs des organisations impliquées, qui entendent également se soutenir mutuellement dans des questions spécifiques. Ensemble, nous serons

L'une des activités principales de la médecine vétérinaire est l'hygiène des denrées alimentaires.

mieux entendus du public, et c'est précisément la raison pour laquelle il vaut la peine de porter le regard sur nos revues respectives, comme nous le faisons à travers cet article.

\section{Référence}

1 Häsler Stephan, 200 ans d'Archives suisses de médecine vétérinaire, ASMV 2016, volume 158, cahier 1, pp. 4-8. Lien: www.gstsvs. $\mathrm{ch} / \mathrm{sat} / 200$. 